\title{
COUPLING EFFICIENCY OF METAMATERIAL MAGNETOOPTICAL INTEGRATED ISOLATOR
}

\section{El-Khozondar/Hala}

Electrical Engineering Department, Islamic University of Gaza, P.O.Box 108, Gaza, Palestinian Authority Lehrstuhl für Messsystem-und Sensortechnik, Theresiensstr. 90, D-80333 München, Germany

El-Khozondar/Rifa

Physics department, Al-Aqsa University, Gaza, Palestinian Authority

Institute of Physical Metallurgy and Metal Physics, Kopernikusstraße 14, 52074 Aachen, Germany

Mohammed M. Shabat

Physics department, Islamic University of Gaza,P.O. Box 108, Gaza, Palestinian Authority

Alexander W. Kock

Lehrstuhl für Messsystem-und Sensortechnik, Theresiensstr. 90, D-80333 München, Germany

\section{Introduction}

Since the spectral stability of lasers degrades in the presence of reflection, nonreciprocal devices such as isolators are crucial to stabilize laser oscillation by eliminating reflected light in optical communication systems. ${ }^{1,2}$ Magnetooptical waveguides are the fundamental components for nonreciprocal waveguide isolators. The main ideas of the magnetooptical waveguides depend on the magnetooptic mode coupling in which the key parameter is the difference of the propagation constants of the coupled modes. ${ }^{3,4}$ Best design of a magnetooptical isolator using channel waveguides is difficult to be considered in three dimensions. Therefore, the effective-index method is used to reduce the three dimensional problem to match the solution of two-dimensional (2-D) problem. ${ }^{5}$ For nonreciprocal integrated optical isolators, the variation of the propagation constant when direction of propagation is reversed, leads to a significant device parameter. The difference $\Delta \beta$ between the phase constants for forward and backward propagation of TM modes is used to quantify the nonreciprocity of a mode. ${ }^{6}$ The nonreciprocal phase shift is produced by asymmetric guiding TM modes in the presence of a dc magnetic field applied transversely to the light propagation direction in the film plane. ${ }^{7}$ Bi-substituted rare-earth ions in polycrystal garnet produce magnetooptical garnet films which have a large Faraday rotation $^{8}$ and a large absorption coefficient at $1.3 \mu \mathrm{m}$ wavelength used for fiber-optic communication. ${ }^{9} \mathrm{~A}$ substantial effort has been made to study nonreciprocal operation of TM modes guided by waveguide with a magetooptic film layer. ${ }^{10-13}$ However, a few attempts have been performed to study the application of metamaterials (MTMs) into nonreciprocal isolators. Physical effects of metamaterials with simultaneously negative values of permittivity and permeability were first investigated theoretically by Veselago. $^{14}$ These metamaterials are also known as left handed media because the electric and magnetic fields form a left-handed set of vectors. The physical awareness of these left handed media was exhibited for a new set of engineered materials named left handed metamaterials. ${ }^{15}$ Such metamaterials have attracted attention in optical systems due to their unusual properties. Kim et al. ${ }^{16}$ have numerically investigated the guided dispersion characteristics of the grounded metamaterial slab waveguide for various metamaterial parameters. The results may have applications in areas of the subwavelength compact waveguiding structures with the improved power-confinement characteristics of the superslow waves. Stress effect on the performance of optical waveguide sensor consists of dielectric slab inserted between metamaterial cladding and metamaterial substrate has been investigated by using numerical calculations. $^{17}$ The role of the MTMs parameters on the guided dispersion characteristic of the waveguide isolator is numerically investigated by introducing several sets of negative permittivity and permeability with their products kept the same. ${ }^{18,19}$

In the present study, we present a new type of an integrated magnetooptical isolator. We considered the film and the cover regions are magnetooptic while the substrate is made of metamaterial. The proposed isolator uses only the TM mode propagating perpendicular to the in-plane magnetization of a planer magnetooptical waveguide. The cut-off thickness of the integrated isolator varies with the propagation direction. The chosen thickness of the proposed isolator yields a big difference between the coupling coefficient for forward and backward propagation. Typically, planner waveguide isolators have three layers: the cover, film, and substrate. In the structure treated in Refs. 18 and 19, the film is the only magnetooptic layer. It would be interesting to see if the noreciprocity is assisted or hindered if the cover region is also magnetooptic. In section II, we inspect waveguide isolator where the cover and the film regions are magnetooptic as well as anisotropic and derive an exact dispersion relation for TM modes. Next, section III is focused on numerical calculations followed by discussion of the results in section IV. 


\section{The Optical Isolator}

A schematic structure of the waveguide is shown in Fig. 1. The waveguide consists of three layers: the cover, the film, and the substrate. The cover and film regions consist of iron garnet films with dielectric tensor $\varepsilon_{i}$ is defined as ${ }^{20}$

$$
\varepsilon_{i}=\left(\begin{array}{ccc}
\varepsilon_{x x i} & 0 & j \varepsilon_{x z i} \\
0 & \varepsilon_{y y i} & 0 \\
-j \varepsilon_{x z i} & 0 & \varepsilon_{z z i}
\end{array}\right),
$$

where $i$ denotes $c$ for the cover and $f$ for the film. The magnetization $M$ is adjusted in the cover and film planes perpendicular to the field propagation direction. All numbers in the dielectric tensor are real. Gyrotropy, the off-diagonal components $\varepsilon_{x z i}$, is the result of magnetization and related to the specific Faraday rotation $\theta_{F}$ by $\left|\varepsilon_{x z i}\right| \approx 2 n_{i}\left|\theta_{F}\right| / k$, where $n_{i}$ is the refractive index, where $i=f$ for the film and $i=c$ for the cladding, and $k=2 \pi / \lambda$ the vacuum wave number with $\lambda$ is the vacuum wavelength. The substrate is filled with MTMs having permittivity $\varepsilon_{s}$ and permeability $\mu_{s}$.

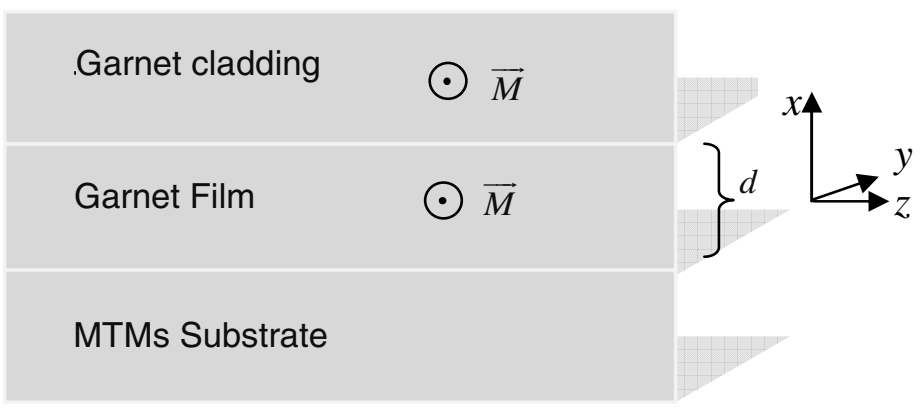

Fig. 1. Basic Geometry of the waveguide isolator.

Consider plan wave propagating in the $z$ direction. The electric and magnetic fields for Transverse Magnetic mode (TM) assume the following oscillating form

$$
\begin{aligned}
& \vec{E}=\left(E_{x}, 0, E_{z}\right) \exp [j(\omega t-\beta z)], \\
& \vec{H}=\left(0, H_{y}, 0\right) \quad \exp [j(\omega t-\beta z)],
\end{aligned}
$$

where $\beta=n_{\text {eff }} k$ denotes the propagation constant, $n_{\text {eff }}$ the effective refractive index, $\omega=c k$ the frequency, and $c$ is the speed of light. The waveguide is assumed homogeneous in $z$-direction, the direction of light propagation, and that all the materials are lossless.

In the film and cladding regions, applying the fields Eq. (2), into Maxwell's equations yields the following dispersion equation:

$$
\frac{d^{2} H_{y i}}{d x^{2}}+\left(k^{2} \varepsilon_{v i}-\beta^{2}\right) H_{y i}=0,
$$

where $i$ denotes $f$ for the film region and $c$ for the cladding region, and $\varepsilon_{v i}=\varepsilon_{z z i}-\frac{\varepsilon_{x z i}^{2}}{\varepsilon_{x x i}}$.

In the calculations, it is assumed that the magnetooptic media are isotropic (i.e. $\varepsilon_{x x i}=\varepsilon_{z z i}$ ). The transverse fields can be calculated from the following equations

$$
\begin{gathered}
H_{y i}=\frac{\omega \varepsilon_{x x i}}{\beta} E_{x i}+\frac{j \varepsilon_{x z i} \omega}{\beta} E_{z i}, \\
E_{z i}=\frac{1}{j \varepsilon_{v i} \omega}\left[\frac{d H_{y i}}{d x}-\frac{\beta \varepsilon_{x z i}}{\varepsilon_{x x i}} H_{y i}\right], \\
E_{x i}=\frac{\beta H_{y i}-\frac{\varepsilon_{x z i}}{\varepsilon_{x x i}} \frac{\partial H_{y i}}{\partial x}}{\varepsilon_{0} \omega \varepsilon_{v i}}
\end{gathered}
$$


The transverse magnetic field in the film which satisfies Eq. (3) is assumed to be,

$$
H_{y f}=B \sin \left(\gamma_{f} x\right)+C \cos \left(\gamma_{f} x\right),
$$

where $\gamma_{f}=\sqrt{k^{2} \varepsilon_{v f}-\beta^{2}}$, and $B$ and $C$ are constants to be found from boundary conditions.

In the cladding, the solution of Eq. (3) is

$$
H_{y c}=D e^{-\gamma_{c}(x-d)}
$$

where $\gamma_{c}=\sqrt{\beta^{2}-k^{2} \varepsilon_{v c}}$ and $D$ is a constant to be calculated using boundary conditions. For the film and the cladding, the corresponding electric field component, $E_{z i}$ is defined by Eq. (5).

In the substrate region, Maxwell's equations for the transverse components of the electric and magnetic field vectors yield the following differential equation:

where $n_{s}=\sqrt{\varepsilon_{s} \mu_{s}}$.

$$
\frac{d^{2} H_{y s}}{d x^{2}}+\left(\beta^{2}-k^{2} n_{s}^{2}\right) H_{y s}=0
$$

$$
H_{y s}=C e^{\gamma_{s} x},
$$

where $\gamma_{s}=\sqrt{\beta^{2}-k^{2} n_{s}^{2}}$, and $C$ is constant to be found using boundary conditions. For the substrate, the corresponding electric field component, $E_{z s}$ is

$$
E_{z s}=\frac{1}{j \omega \varepsilon_{s}} \frac{d H_{y s}}{d x} .
$$

Using the solutions for $H_{y}$ and $E_{z}$ and applying the boundary conditions (continuity of $E_{z}$ and $H_{y}$ ), we obtain the transverse equation:

$$
\gamma_{f} d=\theta_{c}+\theta_{s}+m \pi
$$

where

$$
\begin{gathered}
\theta_{c}=\tan ^{-1}\left(\frac{1}{\gamma_{f}}\left[\frac{\varepsilon_{v f}}{\varepsilon_{v c}}\left(\gamma_{c}+\beta \frac{\varepsilon_{x z c}}{\varepsilon_{x x c}}\right)-\beta \frac{\varepsilon_{x x f}}{\varepsilon_{x x f}}\right]\right), \\
\theta_{s}=\tan ^{-1}\left(\frac{1}{\gamma_{f}}\left[\frac{\varepsilon_{v f}}{\varepsilon_{s}} \gamma_{s}+\beta \frac{\varepsilon_{x x f}}{\varepsilon_{x x f}}\right]\right),
\end{gathered}
$$

and $m$ is an integer, indicates the mode order.

Since linear terms of the propagation constant $\beta$ come in Eqs. (13) and (14), the solution of Eq. (12) is different for the forward direction $\beta^{+}$and the backward direction $\beta^{-}$depending on the sign of $\beta$. The difference $\Delta \beta=\beta^{+}-\beta^{-}$between the forward and backward propagation is calculated numerically. To achieve a large $\Delta \beta$, the permittivity $\left(\varepsilon_{s}\right)$ and the permeability $\left(\mu_{s}\right)$ of the metamaterial substrate are varied by introducing several sets of parameters with their products kept the same, i.e., $\varepsilon_{s} \mu_{s}=4.0$ as used before in Ref. 16.

To measure the confinement of such isolator, the intensity distribution of the fields is calculated. The intensity is normalized to the total intensity $I_{0}$ of the waveguide:

$$
I_{0}=\int_{-\infty}^{+\infty}\left|E_{x}\right|^{2} d x
$$

The coupling efficiency $\eta$ of the optical isolator into a fiber optics which represented by Gaussian profile is determined to estimate the forward and backward losses of the isolator and is give by,

$$
\eta=\frac{\left|\int_{-\infty}^{+\infty} E_{x} \cdot E_{x}^{G} d x\right|^{2}}{\int_{-\infty}^{+\infty}\left|E_{x}\right|^{2} d x \cdot \int_{-\infty}^{+\infty}\left|E_{x}^{G}\right|^{2} d x},
$$

where $E_{x}$ represents the propagating field into the isolator and $E_{x}^{G}$ is the electric field distribution of the Gaussian profile describing a glass fiber and can be expressed as follows,

$$
E_{x}^{G}=E_{x_{0}}^{G} \cdot \exp \left(-\frac{(x-d)^{2}}{2 r^{2}}\right),
$$


where $r$ is the decay width. The Gaussian profile is centered at the film-cover interface, $x=d$ at which the intensity of the isolator is maxima.

\section{Numerical Calculations}

The numerical calculations have been perfumed by selecting typical parameters of magnetooptical material for both the film and the cover. The parameters used in these calculations are: $\varepsilon_{c x x}=4.84, \varepsilon_{f x x}=$ $4.84+\Delta \varepsilon_{x x}, \Delta \varepsilon_{x x}$ is small number of the order of $10^{-3}$ as shown in Ref. $21, k=2 \pi / 1.3 \mu m^{-1}, \varepsilon_{c x z}= \pm 0.005$ where $(+)$ indicates for forward propagation and (-) indicates for backward propagation and $\varepsilon_{f x z}=-\varepsilon_{c x z}$. A large value of $\Delta \varepsilon_{x z}=\varepsilon_{c x z}-\varepsilon_{f x z}$ can be achieved when the absolute values of the components $\varepsilon_{j x z}$ are large and have opposite signs in cover and film. For this purpose, Bi-substitution of polycrystal garnet film is functional. The value of $\varepsilon_{i x z}=0.005$ matches a specific Faraday rotation of $2880 \% \mathrm{~cm}$ at a wavelength of $1.3 \mu \mathrm{m}{ }^{22}$ For the MTM substrate, we chose several sets of $\mu_{s}$ and $\varepsilon_{s}$ with their multiplication kept constant as in Ref. 16. The selected values are presented in Table 1.

Table 1. Combinations of $\varepsilon_{s}$ and $\mu_{s}$ with their product kept constant, i.e., $\varepsilon_{s} \mu_{s}=4$.

\begin{tabular}{|c|c|c|c|c|}
\hline & 1 & 2 & 3 & 4 \\
\hline$\varepsilon_{\mathrm{s}}$ & -8.0 & -4.0 & -2.0 & -1.0 \\
\hline$\mu_{\mathrm{s}}$ & -0.5 & -1.0 & -2.0 & -4.0 \\
\hline
\end{tabular}

\section{Results}

The focus of our study is only the first TM propagated mode which corresponds to $m=1\left(T M_{1}\right)$. The effective refractive index of these $\mathrm{TM}_{1}$ is different for forward and backward propagation. The cut-off thickness of the forward and backward propagation is drawn as a function of the difference $\Delta \varepsilon_{x x}=\varepsilon_{f x x}-$ $\varepsilon_{c x x}$ in Fig. 2 for different sets of $\varepsilon_{s}$ and $\mu_{s}$ for MTM substrate as listed in table 1. The value of $\Delta \varepsilon_{x x}$ is taken to be equals to $10^{-3}$. The cut-off thickness $d^{c+}$ and $d^{c-}$ are of the order of 9.2 and $11.1 \mu \mathrm{m}$ for forward and backward propagation respectively. These values are in good agreement with the diameter of fiber optics.

Fig. 3 illustrates the dependence of the propagation constants $\left(\beta^{+}\right.$, and $\left.\beta^{-}\right)$for forward and backward propagation respectively on the film thickness at the value of $\Delta \varepsilon_{x x}=10^{-3}$. It is shown that the propagation constants depend on the values $\varepsilon_{s}$ and $\mu_{s}$ for the MTM substrate as listed in table 1.

Fig. 4 displays the coupling efficiencies $\eta^{+}$and $\eta^{-}$for forward and backward propagation correspondingly. The coupling coefficients to the isolator are highly different at the cutoff thickness for forward and backward propagation. Moreover, the coupling efficiencies depend on the values of the MTM parameters.

The intensity of the forward propagating $\mathrm{TM}_{1}$ along the film normal is demonstrated in Fig. 5 for variant values of the film thickness in the range of the cut-off thicknesses $d^{c+}$ and $d^{c-}$. The difference $\Delta \varepsilon_{x x}$ is considered to have the value of $10^{-3}$ in this calculation. The intensity distribution attains intensely into the cover region.

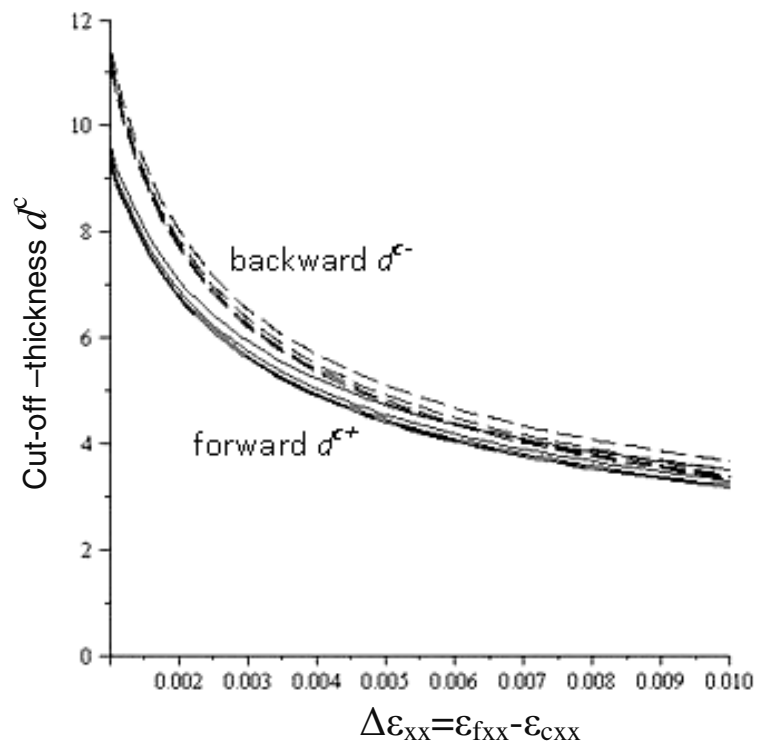

Fig. 2. Change of cut-off thickness with the difference between the components $\varepsilon_{x x}$ of the dielectric tensors of film and cover at different values of $\varepsilon_{s}$ and $\mu_{s}$ with their product kept constant of the value equal to 4 . going from up to down, the values of $\varepsilon_{\mathrm{s}}$ are equal to -8, 4. -2 . and -1 . 


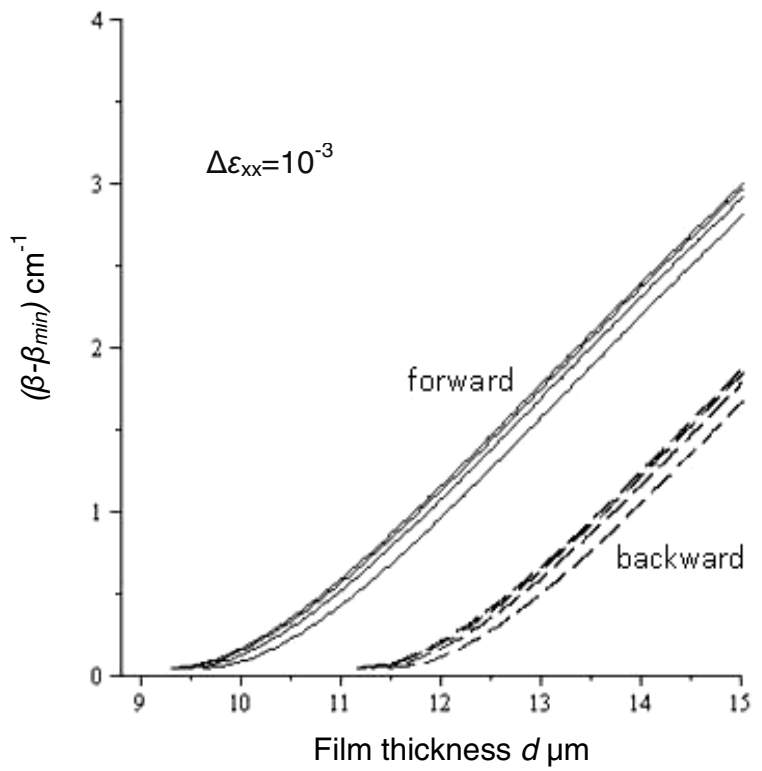

Fig. 3 Dependence of propagation constant of the $\mathrm{TM}_{1}$ mode on film thickness for forward and backward propagation for various values of $\varepsilon_{\mathrm{s}}$ and $\mu_{\mathrm{s}}$. starting from up the values of $\varepsilon_{\mathrm{s}}$ are: $-8,-4,-2$, and -1

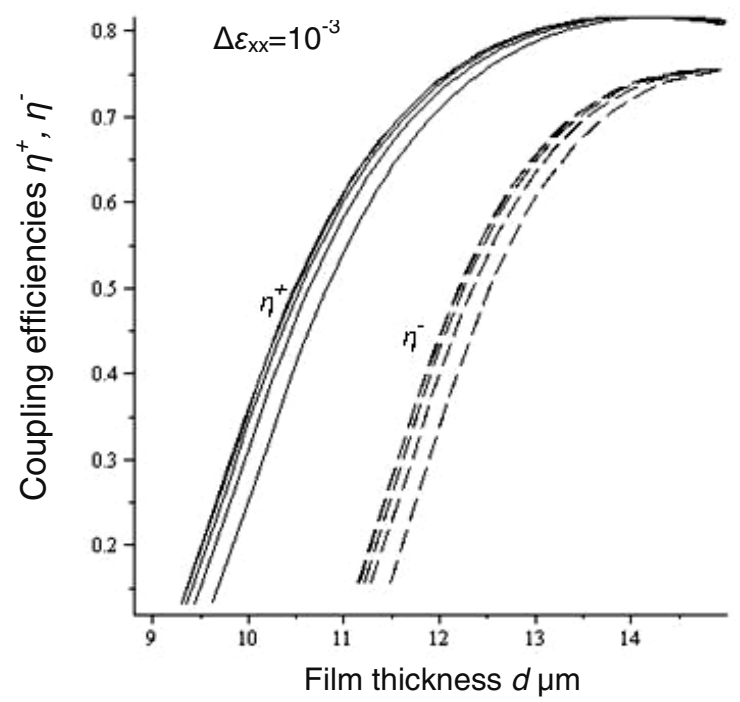

Fig. 4. Simulated coupling efficiency between $\mathrm{TM}_{1}$ mode of the waveguide and a glass fiber which is characterized by a Gaussian profile for different values of $\varepsilon_{\mathrm{s}}$ and $\mu_{\mathrm{s}}$. From below the values of $\varepsilon_{\mathrm{s}}$ are: $-8,-4,-2$, and -1 .

\section{Conclusion}

The cutoff thickness of magnetooptical isolator consists of isotropic magnetooptical materials film and cladding and MTMs substrate is calculated. It is found that the cutoff thickness of the isolator can be tuned by changing the values of $\varepsilon s$ and $\mu \mathrm{s}$. A further step is done by calculating the coupling efficiencies into Gaussian profile for forward and backward propagation. The results exhibit that the coupling efficiencies vary with changing the MTM parameters. The thickness of the isolator is chosen such that a big variation between the coupling efficiencies for forward and backward propagation is produced. The results are encouraging to be applied to the design of various nonreciprocal integrated-optical devices which use the first mode. 


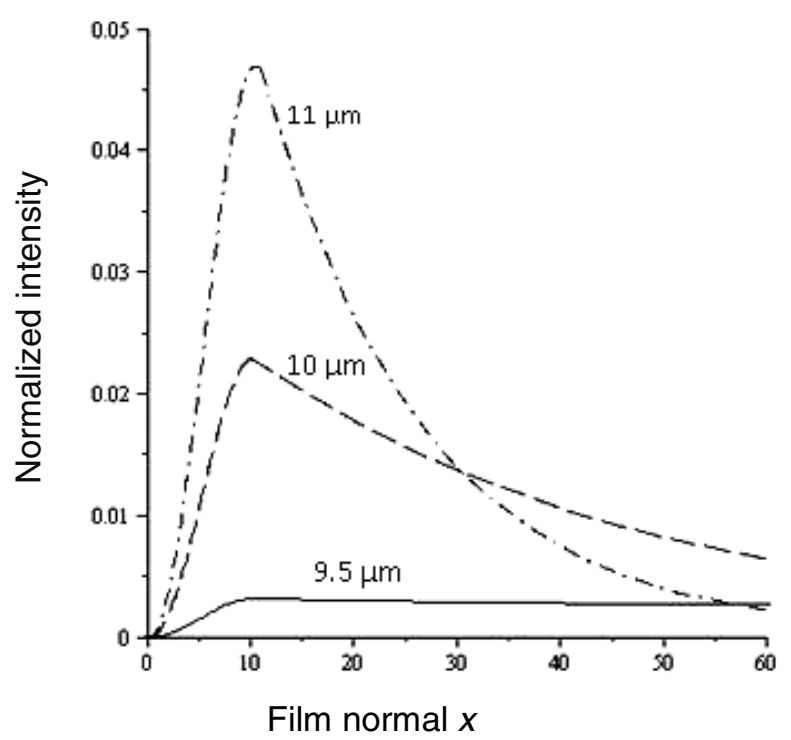

Fig. 5. Difference of the normalized intensity of the $\mathrm{TM}_{1}$ mode along the film normal; parameter is the film thickness at $\varepsilon_{\mathrm{s}}=-4$ and $\mu_{\mathrm{s}}=-1$.

\section{References}

1. K. Ando, T. Okoshi and N. Koshizuka, Appl. Phys. Lett. 53(1), 4-6 (1988).

2. E. Pross, W. Tolksdrof and H. Dammann, Appl. Phys. Lett. 52(9), 682-684(1988).

3. S. Yamamoto and T. Makimoto, J. Appl. Phys. 45(2), 882-888 (1974).

4. H. Damman, E. Pross, G. Rabe and W. Tolksdort and M. Zinke, Appl. Phys. Lett. 49(26), 17551757 (1986).

5. L. A. Zenteno, Opt. Lett. 12(9), 657-659 (1987).

6. C. Chen and A. Kumarswami, Appl. Opt. 25(20), 3664-3670 (1986).

7. H. Yokoi, T. Mizumuto, N. Shinjo, N. Futakushi and Y. Nakano, Appl. Opt. 39(33), 61586164(2000).

8. K. Shingawa, and S.Taniguchi, Jap. J. of Appl. Phys. 13(10), 1663-1664 (1974).

9. T. Hibiya, Y. Morishige and J. Nakashima, Jap. J. of Appl. Phys. 24(10), 1316-1319 (1985).

10. N. Bahlmann, M. Lohmeyer, A. Zhuromskii, H. Dötsch, P. Hertel, Opt. Commun. 161, 330-337 (1999).

11. H. Hemme, H. Dötsch, P. Hertel, Appl. Opt. 29(18), 2741-2744 (1990).

12. M. Shamonin, M. Lohmeyer, P. Hertel, H. Dötsch, Opt. Commun. 131, 37-40 (1996).

13. H. Dötsch, P. Hertel, B. Lührmann, S. Sure, H. P. Winkler and M. Ye+, IEEE Trans. Magn. 28(5), 2979-2984 (1992).

14. V. G. Veselago, Sov. Phys. Uspekhi 10(4), 509- 514 (1968).

15. D. R. Smith, W. J. Padilla, D. C. Vier, S. C. Nemat-Nasser, and S. Schultz, Phys. Rev. Lett. 84 (18), 4184-4187 (2000).

16. K. Y. Kim, Y. K. Cho, and H. Tae, J. Korean Phys. Soc. 49(2), 577-584 (2006).

17. H. J. El-Khozondar, R. J. El-Khozondar and M. M. Shabat, The Islamic University Journal (Series of Natural Studies and Engineering) 16(1), 1-12 (2008).

18. R. J. El-Khozondar, H. J. El-Khozondar and M. M. Shabat, J. Al-Aqsa Unv.12, 35-50 (2008).

19. R .J. El-Khozondar, H .J. El-Khozondar, M. M. Shabat, Proc. SPIE 6987, 69872A1-5 (2008).

20. V. Doorman, J. P. Krumme, C. P. Klages and M. Erman, Appl. Phys. A 34, 223-230 (1984).

21. W. Tolksdrof, H. Dammann, E. Pross, B. Strocka, H. J. Tolle, and P. Willich, J. Cryst. Growth 83, 15-22 (1987).

22. T. Hibiya, Y. Morishige, and J. Nakashima, Jap. J. Appl. Phys. 24, 1316-1319 (1985). 